\title{
Meta-analysis of Psychological Interventions on Breast Cancer Patients' Immune Function
}

\author{
Ling QI \\ School of Health Science and Nursing \\ Wuhan Polytechnic University, \\ Wuhan, China \\ qiolive_23@163.com \\ Hong-ling $\mathrm{ZHANG}^{*}$ \\ School of Health Science and Nursing \\ Wuhan Polytechnic University, \\ Wuhan, China \\ zhlwjb@sina.com
}

\author{
Jing WANG \\ Key Laboratory of Applied Psychology \\ Chongqing Normal University \\ Chongqing, China \\ wangjingsyxy@163.com
}

\begin{abstract}
Objective: To evaluate the efficacy of psychological interventions for improving the immune functioning of breast cancer patients.

Methods: Comprehensive searches of electronic databases from 1990 to 2012, hand searches of reference lists of articles, and contacts with researchers. Meta-analytic approaches were used on synthesized findings. Pre-post and between-group intervention effects on symptoms of cortisol, lymphocytes, natural killer cell counts, and natural killer cell cytotoxicity were assessed, respectively.

Results: Data from 9 controlled trials were analyzed. Significant intervention effect was for improving function of natural killer cell cytotoxicity (pre-post ES=-2.53, betweengroup ES=-3.99). There are limited evidences suggesting psychological interventions effect on other immune parameters. Conclusions: The effect of psychological interventions on immune function in breast cancer patients depends on the features of the immune parameters. The relationship between the psychological intervention effects and immune function will require further study. Future research should identify potential confounding factors associated with psychological interventions effects.
\end{abstract}

Keywords-psychological interventions; breast cancer; immune function; randomized controlled trial; meta-analyses

\section{INTRODUCTION}

Despite the increasing survival rates however, the diagnosis of breast cancer and subsequent treatments mean stressful events for patients. After diagnosis patients deal with anticipatory anxiety over how treatment will affect their bodies, prior to receiving adjuvant therapy. Then they can experience physical complications of treatments, shifts in social support, shifts in their bodies and fear of recurrence and death during and after many treatments such as surgery, radiation, and chemotherapy[1]. According to Biobehavioral Model of Cancer Stress and Disease Course, psychological stress leads to decrease in healthy behaviors, lower quality of life, and weakened immunity, which in turn contribute to poorer disease outcomes[2]. Therefore, identification of interventions to reduce toxicities, distress, and maintain maximal immune competence in breast cancer patients has great therapeutic relevance. In the past few decades, there has been considerable interest in mind-body medicine and complementary therapies within the breast cancer patients[3] Psychological interventions with undergoing cancer treatment has received wide acceptance from both medical staff and patients during the last 20 years [4]. A number of studies have documented that psychological interventions associate with positive psychological effects among breast cancer patients [4]. However, it is still less knows about whether psychological intervention can influence the biological process in breast cancer patients.

In the past 10 year it has been become clear that human breast cancer is immunogenic[5]. Immune system is believed to play an important role in recognizing breast cancer antigens, and in receiving better therapeutic outcomes. Antigen presenting cells, such as natural killer cells, cot toxic-T-cells, Trgulatory cells, and B cells, are all believed to play an important role in the host response against spontaneous tumors[5]. Other work shows the cytokines interleukin-2 (IL-2) and interferon-gamma (IFN- $\gamma$ ) may work together to promote surveillance of tumor cells and may play a role in disease recurrence after treatment[1]. On the other hand, a number of literature shows there is significant relationship between psychological distress and immune function among breast cancer patients. Continuing levels of high stress had been proved that associated with decrements in cellular immune functions that help prevent infectious and metastasis[6,7]. Some have pointed that women diagnosed with breast cancer have significant psychosocial distress that was accompanied by deregulated natural killer cell activity and cytokine production[1]. 
Elevated levels of distress were associated with reduced natural killer cell cytotoxicity[8].Given the close interaction between the nervous, endocrine and immune system, psychological intervention may also improve patients' immune functions [9].

Guided by a psychoneuroimmunologic framework that proposes that psychosocial factors may influence immunologic outcomes via sympathetic nervous system and neuroendocrine pathways [10], it is proposed that psychological interventions can not only bring out positive psychological effects, but also enhance immune functioning. At present, several studies have reported improved immune functioning among psychological interventions participants[11,12]. However, there are inconsistence results about the effectiveness of psychological interventions on breast cancer patients' immune function. Findings of other studies suggested that psychological intervention had no positive effect on immune function[13].These inconsistencies among previous studies are due to the considerable potential confounding variables, including differences in the patients' clinical and demographic characteristics, methodological design, type and duration of psychosocial interventions, and selection of immune variables. In conclusion, it remains unclear whether and to what extent the efficacy of psychological interventions in immune function. Whether the psychological interventions can reliably influence cancer patients? How large is the overall effect size of psychosocial interventions in cancer patients? By combining results across independent researches, meta-analysis can more powerfully draw conclusions.

There are ten meta-analyses examining the effectiveness of various psychological interventions for cancer patient since the year 2000[14-23]. Overall, these meta-analyses report promising result on the effectiveness of psychological interventions for improving quality of life, alleviating anxiety and depression, controlling pain and fatigue. Only one study examined the effectiveness of various psychological interventions for enhancing immune function [22]. Breast cancer is the most common tumor among women worldwide, and the incidence has been increasing since the 1970s [24]. However, we are aware of no study that has specifically addressed the effectiveness of psychological intervention in breast cancer patients' immune functioning. The question of whether psychological intervention could have an effect on the immune function of breast cancer patients has yield inconsistent results as mentioned above. Therefore, our objective in this metaanalysis was to evaluate the immunological effects of psychological interventions in breast cancer patients. We wanted to assess the effects on those parameters of cellmediated immunity and natural cytotoxicity, and their associated regulatory cytokines, which are believed to play a crucial role in breast cancer progression and treatment. The review primarily analyses the overall pre-post-effect sizes (ESs) of psychological interventions for immune parameters, secondarily, between-group ESs for different control conditions.

\section{METHOR}

\section{A. Database and Literature Search Strategy}

Experiment studies measuring immune function in breast cancer patients were reviewed for potential inclusion in this meta-analysis. We performed electronic searches in PsychInfo (from 1990 to 30 May 2012), PubMed((from 1950 to 30 May 2012),Web of Science(from 1986 to 30 May 2012), and Google. We used combinations of the following terms: psychotherapy, psychological interventions, immune function, relaxation, hypnosis, cognitive behavioral therapy, biofeedback, disclosure, breast cancer, and psychoneuroimunnology. The electronic search was set to accept studies published in English. In addition, we manually searched the reference lists of literature reviews and meta-analyses on the psychological intervention of cancer symptoms. Supplementary information was sometimes obtained directly from the authors or from other published reports. The studies found were then downloaded into NoteExpress and duplicates were deleted. The search procedures yielded a pool of 101 studies.

As the focus of this meta-analysis was the effectiveness of psychological interventions, studies not using any psychological interventions were excluded. As Cawley(1993) pointed psychological interventions mean interventions designed to improve psychological and/or physical well-being through the modification of emotion, cognition, or behavior. In this paper, psychological interventions were broadly defined and included any intervention containing some kind of or combination of education, skill training, relaxation, disclosure, etc.

Other inclusion criteria included the use of (1) data collected on at least one biologic outcomes related to immune functioning (i.e., natural killer cell activity, CD4), (2) sufficient data to allow the calculation of effect size,(3)randomization design,(4) between-group design.

All results (initially identified 112 studies) were assessed at either title, abstract, or by reading the full paper to determine whether the study met the inclusion criteria. When we applied these criteria, 30 studies were excluded from the pool for using nonexperimental methods, 23were excluded for insufficient quantitative data, 8 were excluded for outcomes of interest not assessed, 9 were excluded for interventions of interest not tested, and 33 other studies were subsequently excluded because the participants were not beast cancer patients. We finally selected 9 studies for the present meta-analysis (the selection process see figure 1). 


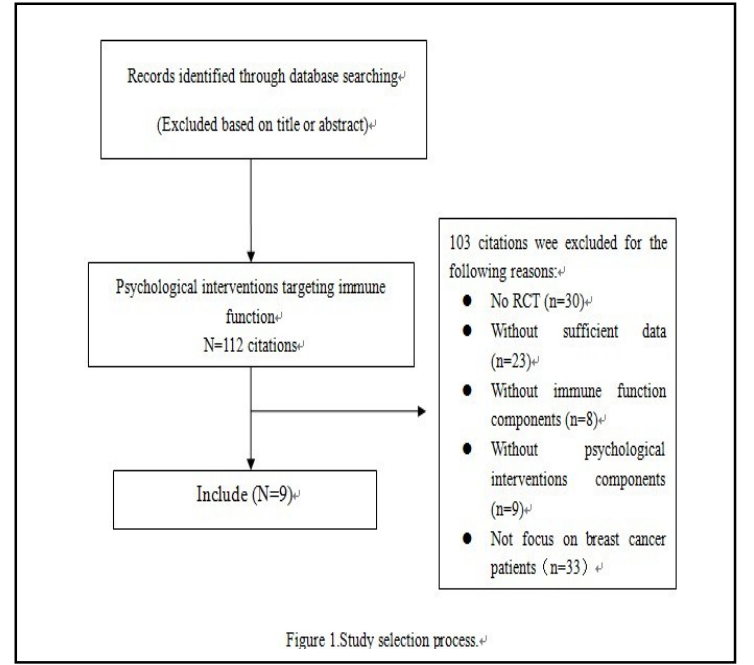

\section{B. Data Abstraction}

Two trained reviewers extracted information from eligible reports independently. Using standardized coding forms, each trail was coded for general information (e.g., authors'year of the published report, country), characteristics of participants (e.g., number of participants, breast cancer stage); characteristics of intervention (e.g., content, frequency, deliverer and duration), and outcomes (e.g., type).We also coded variables related to methodological quality of the trials: assignment method, type of control group, sample size, and differential retention. Disagreements between reviewers were resolved by consensus following a joint review of the articles. Data on effect size that could not be directly abstracted were recalculated, where possible.

\section{Analytical Approach}

Because considerable differences among trials, we applied the following rules for estimating the overall intervention effect. Separate analyses were conducted for each of the following four biological markers of immune function (cytotoxicity, cytokines, cortisol, and leukocyte).We calculated effect size by using data from adjusted models reported by the authors because baseline differences and potential confounding variables are typically controlled in true experiment study. For trials that reported multiple assessments, we selected the first post-intervention assessment for evaluating intervention effects. We calculated effect size for each independent sample if a trial reported data on each sample (e.g., men and women) separately. These decision rules have been previously applied in a meta-analytic review of cognitive-behavioral interventions on HIV-positive persons' mental health and immune functioning [25]. Otherwise, for trails assessed more than one psychological interventions relative to a single control group, we calculate effect size for each interventions. To avoid overestimate counting, the control group sample size was halved and rounded down to the nearest whole number [26].

\section{Statistical Analysis}

Data were analyzed with Stata 10.The effect size indictor for this meta-analysis was the weight mean difference (WMD). For each meta-analysis, an overall effect size was estimated by weighting the average effect size for each study according to sample size and then pooling across studies. Each marks of immune functioning in each condition at each time point were extracted to calculate ingroup effect size and between-group effect size. Homogeneity tests were also conducted to test whether a set of effect sizes could be considered as a sample form a single underlying effect size, using the Cochran's Q [27], a p-value of $<0.10$ for the chi-squared test for heterogeneity was considered significant.

\section{RESULT}

\section{A. Description of Trails}

Nine trials, published between 1994 and 2008, met the inclusion criteria. Table 1 provides a summary of trial characteristics (sample, intervention et al.).Four were conducted in the United State, two in Sweden, two in Canada, and one in Germany. The mean age of participants was 51.47 (two trails did not report the information on the age of participants).With respect to the psychological interventions, the median number of intervention sessions was 10 sessions over a 10 -week period, the majority trails delivered in a group format $(n=7)$. The majority studies included components of cognitive restructuring techniques $(\mathrm{n}=6)$, stress management skills training including relaxation, guided imagery techniques $(n=6)$, and coping skill training were included in fewer studies $(n=3)$.One study helped participants increase social support.

TABLE1. Characteristics of Trails Included in the Meta-Analyses

\begin{tabular}{|c|c|}
\hline Variable & $\begin{array}{c}\text { Number of trails or } \\
\text { mean(SE)/median(range) }\end{array}$ \\
\hline \multicolumn{2}{|l|}{ Country where trial was conducted } \\
\hline United States & 4 \\
\hline Canada & 2 \\
\hline Sweden & 2 \\
\hline Germany & 1 \\
\hline Age $^{\text {a }}$ & $51.47(4.73)$ \\
\hline \multicolumn{2}{|l|}{ Intervention characteristics } \\
\hline Cognitive restructuring techniques & 6 \\
\hline Coping skill training & 3 \\
\hline Stress management skills training & 6 \\
\hline Social support & 1 \\
\hline \multicolumn{2}{|l|}{ Unit of intervention delivery ${ }^{b}$} \\
\hline Group & 7 \\
\hline Individual & 1 \\
\hline \multicolumn{2}{|l|}{ Intervention deliverer ${ }^{c}$} \\
\hline Professional psychologist or psychiatrist & 3 \\
\hline Trained research staff & 3 \\
\hline Number of intervention sessions & $\mathrm{Mdn}=10($ range 4 to 18$)$ \\
\hline
\end{tabular}

\section{B. Methodological Quality of the Trails}

All trails randomly assigned participants to intervention and control group. The outcomes were assessed 
immediately after the intervention in 7 and the median overall retention was $100 \%$ ( range: $81.1 \%$ to $100 \%$ ). Four trials conducted follow-up assessments ranging from 2 to 12 months $(\mathrm{M} d \mathrm{n}=6$ months) after the completion of an intervention. All trials used the intent-to-treat approach for data analysis.

\section{Intervention Efficacy Compared with Pre-intervention}

As shown in table2, the aggregated effect sizes indicate that the intervention significantly improved natural killer cell cytotoxicity $(\mathrm{ES}=-2.53)$ and lymphocytes (ES $=0.36)$. However, the aggregated effect sizes for cortisol $(\mathrm{ES}=0.08)$ and natural killer counts $(\mathrm{ES}=0.004)$ were not significant. The results of the homogeneity tests indicated heterogeneity among trials reporting the following outcomes: nature killer cell cytotoxicity, cortisol, and lymphocytes. However,there is no evidence of homogeneity in trials reporting natural killer cell cytotoxicity. Additional sensitivity tests did not reveal any single trial that exerted influence on the overall effect size for each outcome.

TABLE 2. EFFECT SIZE, 95\% CONFIDENT INTERVALS, AND HOMOGENEITY TESTS FOR IMMUNE FUNCTION IN BREAST CANCER PATIENTS

\begin{tabular}{|c|c|c|c|c|c|c|}
\hline & $\begin{array}{l}\text { No.of } \\
\text { effects }\end{array}$ & ES & $\begin{array}{c}\mathrm{Z} \\
\text { score }\end{array}$ & $\begin{array}{c}p \\
\text { value }\end{array}$ & $\mathbf{Q}$ & $\begin{array}{c}P \\
\text { value } \\
\text { for } \\
\mathbf{Q} \\
\end{array}$ \\
\hline Cortisol & 2 & 0.08 & 0.74 & 0.46 & 2.8 & 0.1 \\
\hline Lymphocytes & 3 & -0.36 & 3.02 & 0.003 & 5.54 & 0.06 \\
\hline $\begin{array}{l}\text { Natural killer } \\
\text { cell counts }\end{array}$ & 4 & 0.004 & 0.17 & 0.867 & 25.82 & 0.00 \\
\hline $\begin{array}{l}\text { Natural killer } \\
\text { cell } \\
\end{array}$ & 4 & -2.53 & 2.05 & 0.04 & 4.66 & 0.198 \\
\hline
\end{tabular}

\section{Intervention Efficacy Compared with Control Groups}

As shown in Table3, the aggregated effect sizes indicate that the intervention significantly improved nature killer cell cytotoxicity $(\mathrm{ES}=-0.49)$. However, the aggregated effect sizes for cortisol $(\mathrm{ES}=0.15)$, lymphocytes $(\mathrm{ES}=-0.02)$, and natural killer counts $(\mathrm{ES}=0.10)$ were not significant. The results of the homogeneity tests indicated heterogeneity among trials reporting the following outcomes: nature killer cell cytotoxicity, cortisol, lymphocytes, and natural killer counts. Additional sensitivity tests did not reveal any single trial that exerted influence on the overall effect size for each outcome.

TABLE 3. EFFECT SIZE, 95\% CONFIDENT INTERVALS, AND HOMOGENEITY TESTS FOR IMMUNE FUNCTION IN BREAST CANCER PATIENTS

\begin{tabular}{lcccccc}
\hline & $\begin{array}{c}\text { No.of } \\
\text { effects }\end{array}$ & ES & $\begin{array}{c}\mathbf{Z} \\
\text { score }\end{array}$ & $\begin{array}{c}\boldsymbol{P} \\
\text { value }\end{array}$ & $\mathbf{Q}$ & $\begin{array}{c}\boldsymbol{P} \\
\text { value } \\
\text { for Q }\end{array}$ \\
\hline Cortisol & 3 & -0.07 & 0.64 & 0.52 & 2.7 & 0.26 \\
$\begin{array}{l}\text { Lymphocytes } \\
\begin{array}{l}\text { Natural killer } \\
\text { cell counts }\end{array}\end{array}$ & 4 & 0.03 & 0.37 & 0.72 & 5.41 & 0.14 \\
$\begin{array}{l}\text { Natural killer } \\
\text { cell cytotoxicity }\end{array}$ & 4 & 0.04 & 1.79 & 0.07 & 1.45 & 0.84 \\
\end{tabular}

\section{DISCUSSION}

In the present study, we analyzed the effectiveness of psychological interventions on immune function of breast cancer patients. In the present meta-analysis, our focus was on the random controlled trails. Studies with a crosssectional, group-comparison, or semi -experimental design were not included, because the do not allow for making inferences about relationship.

Only 4 studies provided adequate data to assess psychological interventions efficacy on natural killer cell cytotoxicity. The pooled results showed that, adjuvant psychological interventions were more effective in improving natural killer cell cytotoxicity than treatment as usual or being on a waiting list $(\mathrm{ES}=-3.99)$. The pre-post-ES for natural killer cell cytotoxicity was also statistically significant $\quad(E S=-2.53)$ Statistically significant improvements in natural killer cell cytotoxicity were report in three studies[28-30].Despite statistical nosignificant in the Lindemalm and colleague's studies, all point estimates were in favor of psychological interventions, which suggests that psychological interventions have significant effect on improving natural killer cell cytotoxicity of breast cancer patients.

The pooled results of 4 studies examining the effects psychological interventions on lymphocytes showed increasing lymphocytes ( $\mathrm{ES}=-0.36$, for pre- and postinterventions).However the between-group difference in ES with control groups was not statistically significant. This divergent result indicates that psychological interventions have a nonsignificant effect on lymphocytes, and the significant difference between pre- and post-interventions may be induced by other influence factors which need for more research. There were no statistically changes in cortisol and natural killer cell counts as results of psychological interventions trails included in our metaanalyses.

A noteworthy feature of trials included in our review was the wild variability in study interventions. The diversity in psychological interventions is not surprising, given the lack of consensus on the optimal psychological intervention for the breast cancer patients. Conversely, the wide variety in study outcomes is surprising. This variation precluded making overall conclusions regarding the relative effectiveness of psychological intervention difficult by pooling studies. Moreover, data are lacking to examine the effect of psychological intervention on immune function and rehabilitative outcomes in the long term.

This meta-analysis yielded limited evidence that psychological interventions can improve immune function of breast cancer patients. The results suggest that psychological interventions can modulate certain features of the immune response of breast cancer patients. Natural killer cell cytotoxicity was the only outcome to change across two different compared contexts. Natural killer system plays an important role in immuno-surveillance against tumor cells, and the most commonly immunological indicator of disease progression 13. Bower (2003) study has shown that stress correlate with lower levels of natural killer cell cytotoxicity, 
and high levels of natural killer cell cytotoxicity co-vary with a better prognosis and overall survival in many different cancers. Although the preliminary results are promising, the findings are considerably narrower than might have been anticipated. The effect sizes for other enumerative immune function parameters (cortisol, natural killer cell counts, and lymphocytes) were not statistically different from zero. This result may be due to the following reasons. First, the immune system is responsive to psychological interventions but it may not function immediately after interventions[31]. Some researches pointed the immune changes may persist months after the formal intervention has ended[22].Second, short-term interventions may be insufficient to yield improvement for immune function. For example, one study included in this meta-analysis conducted a randomized study comparing "support group program" with a usual treatment control in women with breast cancer. The "support group program" was administered in 4 days [32]. There were no group differences on immune parameters. The limitation in duration time of support program may be the important reason for this result. Third, the psychological interventions in primary studies included certain multiple treatments, which make it difficult to determine which treatment was responsible for the changes in immune function of patients.

Several limitations of the current review should be recognized. The main limitation of the present metaanalyses was the small number of trails, which may weaken conclusions that can be drawn from the review. As such, those comparisons should be viewed with caution, the inclusion of unpublished data and studies published in languages other than English may also have influenced effect sizes. Another limitation of our review was it may include poor quality of the primary data, although only random control trails were included. For example, some studies examined the effects of psychological interventions with small sample size (such as Billhult et al., 2008), which limit the power for detecting statistically significant changes and the external validity of the findings of this meta-analysis.

\section{CONCLUSIONS}

Despite these limitations, to our knowledge this is the first meta-analysis to examine the effect of psychological interventions on immune function in breast cancer patients. Our meta-analyses suggest that effect of psychological interventions on immune function in breast cancer patients depends on the features of the immune parameters. The relationship between the psychological intervention effects and immune function will require further study. Future research should identify potential confounding factors associated with psychological interventions effects.

\section{REFERENCES}

[1] M. H. Antoni, S. Lechner, A. Diaz, S. Vargas, H. Holley, K. Phillips, B. McGregor, C. S. Carver, and B. Blomberg, "Cognitive behavioral stress management effects on psychosocial and physiological adaptation in women undergoing treatment for breast cancer," BRAIN BEHAVIOR AND IMMUNITY, vol. 23, pp. 580-591, 2009.

[2] B. L. Andersen, L. M. Thornton, C. L. Shapiro, W. B. Farrar, B. L. Mundy, H. C. Yang, and W. E. Carson, "Biobehavioral, Immune, and
Health Benefits following Recurrence for Psychological Intervention Participants," CLINICAL CANCER RESEARCH, vol. 16, pp. 32703278, 2010.

[3] L. E. Carlson, Z. Ursuliak, E. Goodey, M. Angen, and M. Speca, "The effects of a mindfulness meditation-based stress reduction program on mood and symptoms of stress in cancer outpatients: 6month follow-up," SUPPORTIVE CARE IN CANCER, vol. 9, pp. $112-123,2001$

[4] W. H. Redd, G. H. Montgomery and K. N. DuHamel, "Behavioral intervention for cancer treatment side effects," JOURNAL OF THE NATIONAL CANCER INSTITUTE, vol. 93, pp. 810-823, 2001.

[5] M. L. Disis and H. K. Lyerly, "Global role of the immune system in identifying cancer initiation and limiting disease progression," JOURNAL OF CLINICAL ONCOLOGY, vol. 23, pp. 8923-8925, 2005 .

[6] B. L. Andersen, W. B. Farrar, D. Golden-Kreutz, L. A. Kutz, R. MacCallum, M. E. Courtney, and R. Glaser, "Stress and immune responses after surgical treatment for regional breast cancer," JOURNAL OF THE NATIONAL CANCER INSTITUTE, vol. 90, pp. 30-36, 1998

[7] B. L. Andersen, J. K. Kiecolt-Glaser and R. Glaser, "A biobehavioral model of cancer stress and disease course.," American Psychologist, vol. 49, p. 389, 1994.

[8] S. K. Lutgendorf, A. K. Sood, B. Anderson, S. McGinn, H. Maiseri, M. Dao, J. I. Sorosky, K. De Geest, J. Ritchie, and D. M. Lubaroff, "Social support, psychological distress, and natural killer cell activity in ovarian cancer," JOURNAL OF CLINICAL ONCOLOGY, vol. 23, pp. 7105-7113, 2005 .

[9] M. Schedlowski, C. Jung, G. Schimanski, U. Tewes, and H. Schmoll, "Effects of behavioral intervention on plasma cortisol and lymphocytes in breast cancer patients: An exploratory study," Psycho-Oncology, vol. 3, pp. 181--187, 1994.

[10] C. Y. Fang, D. K. Reibel, M. L. Longacre, S. Rosenzweig, D. E. Campbell, and S. D. Douglas, "Enhanced Psychosocial Well-Being Following Participation in a Mindfulness-Based Stress Reduction Program Is Associated with Increased Natural Killer Cell Activity," JOURNAL OF ALTERNATIVE AND COMPLEMENTARY MEDICINE, vol. 16, pp. 531-538, 2010.

[11] B. L. Andersen, W. B. Farrar, D. M. Golden-Kreutz, R. Glaser, C. F. Emery, T. R. Crespin, C. L. Shapiro, and W. E. Carson, "Psychological, behavioral, and immune changes after a psychological intervention: A clinical trial," JOURNAL OF CLINICAL ONCOLOGY, vol. 22, pp. 3570-3580, 2004.

[12] D. H. Kang, T. McArdle, N. J. Park, M. T. Weaver, B. Smith, and J. Carpenter, "Dose Effects of Relaxation Practice on Immune Responses in Women Newly Diagnosed With Breast Cancer: An Exploratory Study," ONCOLOGY NURSING FORUM, vol. 38, p. 380-380, 2011

[13] T. Hosaka, Y. Tokuda, Y. Sugiyama, K. Hirai, and T. Okuyama, "Effects of a structured psychiatric intervention on immune function of cancer patients," Tokai journal of experimental and clinical medicine, vol. 25, pp. 183--188, 2000.

[14] K. Tatrow and G. H. Montgomery, "Cognitive behavioral therapy techniques for distress and pain in breast cancer patients: A metaanalysis," JOURNAL OF BEHAVIORAL MEDICINE, vol. 29, pp. 1727, 2006.

[15] R. L. Osborn, A. C. Demoncada and M. Feuerstein, "Psychosocial interventions for depression, anxiety, and quality of life in cancer survivors: Meta-analyses," INTERNATIONAL JOURNAL OF PSYCHIATRY IN MEDICINE, vol. 36, pp. 13-34, 2006.

[16] S. S. Gorin, P. Krebs, H. Badr, E. A. Janke, H. Jim, B. Spring, D. C. Mohr, M. A. Berendsen, and P. B. Jacobsen, "Meta-Analysis of Psychosocial Interventions to Reduce Pain in Patients With Cancer," JOURNAL OF CLINICAL ONCOLOGY, vol. 30, pp. 539-547, 2012.

[17] B. Rehse and R. Pukrop, "Effects of psychosocial interventions on quality of life in adult cancer patients: meta analysis of 37 published controlled outcome studies," PATIENT EDUCATION AND COUNSELING, vol. 50, pp. 179-186, 2003.

[18] E. C. Devine, "Meta-analysis of the effect of psychoeducational interventions on pain in adults with cancer,", 2003, pp. 75--89. 
[19] G. Smedslund and G. I. Ringdal, "Meta-analysis of the effects of psychosocial interventions on survival time in cancer patients," JOURNAL OF PSYCHOSOMATIC RESEARCH, vol. 57, pp. 123-131, 2004.

[20] S. Schneider, A. Moyer, S. Knapp-Oliver, S. Sohl, D. Cannella, and V. Targhetta, "Pre-intervention distress moderates the efficacy of psychosocial treatment for cancer patients: a meta-analysis," JOURNAL OF BEHAVIORAL MEDICINE, vol. 33, pp. 1-14, 2010.

[21] P. B. Jacobsen, K. A. Donovan, S. T. Vadaparampil, and B. J. Small, "Systematic review and meta-analysis of psychological and activitybased interventions for cancer-related fatigue," HEALTH PSYCHOLOGY, vol. 26, pp. 660-667, 2007.

[22] G. E. Miller and S. Cohen, "Psychological interventions and the immune system: A meta-analytic review and critique," HEALTH PSYCHOLOGY, vol. 20, pp. 47-63, 2001.

[23] W. Linden and A. Girgis, "Psychological treatment outcomes for cancer patients: what do meta-analyses tell us about distress reduction?" PSYCHO-ONCOLOGY, vol. 21, pp. 343-350, 2012.

[24] O. Beiki, P. Hall, A. Ekbom, and T. Moradi, "Breast cancer incidence and case fatality among 4.7 million women in relation to social and ethnic background: a population-based cohort study," BREAST CANCER RESEARCH, vol. 14, 2012.

[25] N. Crepaz, W. F. Passin, J. H. Herbst, S. M. Rama, R. M. Malow, D. W. Purcell, and R. J. Wolitski, "Meta-analysis of cognitive-behavioral interventions on HIV-positive persons' mental health and immune functioning," HEALTH PSYCHOLOGY, vol. 27, pp. 4-14, 2008.

[26] B. Gardner, J. Wardle, L. Poston, and H. Croker, "Changing diet and physical activity to reduce gestational weight gain: a meta-analysis," OBESITY REVIEWS, vol. 12, pp. E602-E620, 2011.

[27] J. E. Bower, M. E. Kemeny, S. E. Taylor, and J. L. Fahey, "Finding positive meaning and its association with natural killer cell cytotoxicity among participants in a bereavement-related disclosure intervention," ANNALS OF BEHAVIORAL MEDICINE, vol. 25, pp. $146-155,2003$.

[28] C. A. Lengacher, M. P. Bennett, L. Gonzalez, D. Gilvary, C. E. Cox, A. Cantor, P. B. Jacobsen, C. Yang, and J. Djeu, "Immune responses to guided imagery during breast cancer treatment," BIOLOGICAL RESEARCH FOR NURSING, vol. 9, pp. 205-214, 2008.

[29] A. Billhult, C. Lindholm, R. Gunnarsson, and E. Stener-Victorin, "The effect of massage on cellular immunity, endocrine and psychological factors in women with breast cancer - A randomized controlled clinical trial," AUTONOMIC NEUROSCIENCE-BASIC \& CLINICAL, vol. 140, pp. 88-95, 2008

[30] J. Savard, S. Simard, H. Ivers, and C. M. Morin, "Randomized study on the efficacy of cognitive behavioral therapy for insomnia secondary to breast cancer, part II: Immunologic effects," JOURNAL OF CLINICAL ONCOLOGY, vol. 23, pp. 6097-6106, 2005.

[31] F. I. Fawzy, M. E. Kemeny, N. W. Fawzy, R. Elashoff, D. Morton, N. Cousins, and J. L. Fahey, "A structured psychiatric intervention for cancer patients: II. Changes over time in immunological measures," Archives of General Psychiatry, vol. 47, p. 729, 1990.

[32] C. Lindemalm, F. Mozaffari, A. Choudhury, H. Granstam-Bjorneklett, M. Lekander, B. Nilsson, M. L. Ojutkangas, A. Osterborg, L. Bergkvist, and H. Mellstedt, "Immune response, depression and fatigue in relation to support intervention in mammary cancer patients," SUPPORTIVE CARE IN CANCER, vol. 16, pp. 57-65, 2008. 